\title{
Получение второй гармоники излучения субтерагерцовых гиротронов при удвоении частоты в InP: Fe и ее использование для магнитоспектроскопии полупроводниковых структур
}

\author{
(C) В.В. Румянцев ${ }^{1}$, К.В. Маремьянин ${ }^{1}$, А.П. Фокин ${ }^{2}$, А.А. Дубинов ${ }^{1}$, В.В. Уточкин ${ }^{1}$, М.Ю. Глявин ${ }^{2}$, \\ Н.Н. Михайлов ${ }^{3}$, С.А. Дворецкий ${ }^{3}$, С.В. Морозов ${ }^{1}$, В.И. Гавриленко ${ }^{1}$ \\ ${ }^{1}$ Институт ффизики микроструктур Российской академии наук, \\ 603950 Нижний Новгород, Россия \\ ${ }^{2}$ Институт прикладной фризики Российской академии наук, \\ 603950 Нижний Новгород, Россия \\ ${ }^{3}$ Институт фризики полупроводников Сибирского отделения Российской академии наук, \\ 630090 Новосибирск, Россия \\ E-mail: rumyantsev@ipm.sci-nnov.ru
}

Поступила в Редакцию 24 апреля 2019 г.

В окончательной редакции 29 апреля 2019 г.

Принята к публикации 29 апреля 2019 г.

Обсуждается возможность получения интенсивного терагерцового излучения за счет решеточной нелинейности второго порядка в кристаллах фосфида индия, легированного железом. В качестве источника излучения рассматриваются гиротроны субтерагерцового диапазона. Показано, что эффективность удвоения частоты может достигать 3\%, что открывает возможность создания нового поколения источников излучения терагерцового диапазона частот. Показана возможность использования преобразованного излучения для магнитоспектроскопии полупроводниковых наноструктур с квазидираковским законом дисперсии.

Ключевые слова: гиротроны, удвоение частоты, терагерцовое излучение, фосфид индия, нелинейность второго порядка.

DOI: $10.21883 /$ FTP.2019.09.48132.15

\section{1. Введение}

В последние десятилетия в мире наблюдается беспрецедентный рост активности в так называемом терагерцовом (ТГц) диапазоне электромагнитного спектра. Гигантский разворот работ по исследованию возможности использования ТГц излучения в разнообразнейших и весьма важных применениях позволяет сказать, что сегодня этот диапазон оказался на переднем крае исследований и разработок (см., например, [1-3]). В ТГц диапазоне лежат вращательные спектры многих органических молекул включая колебания биологически важных коллективных мод ДНК и белков, а также фононные резонансы кристаллических решеток, что позволяет развивать новые методы спектроскопии биологических и полупроводниковых структур. Особую привлекательность представляет использование ТГц излучения для диагностики и терапии в биологии и медицине. В отличие от рентгена это излучение является неионизирующим и не вызывает повреждение тканей, что позволяет проводить безвредную для человека диагностику, в том числе раковых опухолей и ожогов.

Сложность создания эффективных терагерцовых источников связана с тем, что в терагерцовом диапазоне плохо применимы хорошо разработанные методы генерации излучений оптического и микроволнового диапазонов. Отдельной задачей является создание узкополосных источников интенсивного ТГц излучения. Мощными источниками терагерцового излучения, основанными на переносе носителей заряда, являются синхротроны и лазеры на свободных электронах, однако дороговизна и размеры препятствуют их широкому использованию даже для чисто научных приложений. Классические приборы вакуумной электроники, такие как лампа бегущей волны и лампа обратной волны, с трудом достигают частотного рубежа в 1 ТГц, и мощность их излучения в этом диапазоне не превышает 1 мВт. Трудности в повышении рабочей частоты подобных вакуумных источников связана со сложностями в изготовлении мелкомасштабной „замедляющей“ системы [4]. В гироприборах частота излучения ограничена величиной магнитного поля (для генерации излучения с частотой 1 ТГц на основной гармонике требуется поле $\sim 38$ Тл) и необходимостью селекции при работе на гармониках гирочастоты, которая, как правило, требует сложных технических решений. С другой стороны, так как эквивалентная температура излучения частотой 1 ТГц составляет всего $47.6 \mathrm{~K}$, в лазерах тепловая релаксация уровней при комнатной температуре приводит к быстрому уничтожению инверсии. Так, квантово-каскадные лазеры (ККЛ), занимающие лидирующие позиции среди компактных полупроводниковых источников в среднем инфракрасном (ИК) диапазоне, на терагерцовых частотах работают только в условиях криогенного охлаждения. На сегодняшний день наибольшие достигнутые рабочие температуры ТГц ККЛ составляют 117 K в непрерывном 
режиме [5] и $199.5 \mathrm{~K}$ при импульсной накачке [6], а мощность излучения достигает 230 мВт в непрерывном режиме [7]. Газовые лазеры работают при комнатной температуре, однако доступны лишь для некоторого набора частот $[8,9]$.

Основные методы генерации терагерцового излучения в полупроводниковых структурах сводятся к оптическому выпрямлению импульсов коротковолнового излучения (как правило, фемтосекундных Тi:Sa-лазеров $[10,11])$, генерации разностной частоты при использовании в качестве накачки параметрических генераторов света [12,13], а также инициации быстрых переходных процессов при возбуждении полупроводника коротким оптическим импульсом [14]. Источники терагерцового излучения, созданные на основе фемтосекундных лазеров, обладают чрезвычайно широким спектром излучения ( 1 ТГц), что не всегда приемлемо. Кроме того, мощность выходного излучения во всех перечисленных методах невысока [14]. На данный момент для генерации терагерцового излучения методом оптического выпрямления, как правило, используются кристаллы $\mathrm{ZnTe}$ [11], $\mathrm{GaP}$ [12,13], $\mathrm{LiNbO}_{3}$ [10]. Для всех характерны довольно большие потери в терагерцовом диапазоне, ввиду чего эффективность преобразования даже в рекордных режимах приближается лишь к $10^{-3}$ [10], а в типичных случаях составляет величины на порядок меньше.

Одним из способов получения интенсивного терагерцового излучения является умножение частоты излучения за счет различных типов нелинейности в полупроводниковых структурах. Генерация третьей гармоники в германии и кремнии $n$-типа проводимости достаточно хорошо изучена $[15]$, в том числе при умножении частоты излучения гиротронов [16,17]. Так, в работе [16] показана генерация третьей гармоники с эффективностью $0.05 \%$ при возбуждении гиротроном с частотой 0.07 ТГц, а в работе [17] - гиротроном с частотой 0.118 ТГц и с эффективностью преобразования $0.07 \%$. В этих работах использовалась нелинейность третьего порядка, которая преимущественно является электронной. Как известно, генерация второй гармоники за счет решеточной нелинейности второго порядка возможна только в кристаллах, в которых отсутствует центр инверсии. Решеточная нелинейность изучалась в арсениде галлия: в качестве источника излучения использовался газовый $\mathrm{CH}_{3} \mathrm{~F}$-лазер с частотами в диапазоне 0.6-1.7 ТГц [18], а также лазер на свободных электронах [19]. Необходимо отметить, что нелинейные свойства материалов $\mathrm{A}^{\mathrm{III}} \mathrm{B}^{\mathrm{V}}$ довольно слабо изучены в дальней ИК области, где существенна частотная дисперсия нелинейного коэффициента второго порядка. По нашим сведениям, имеется лишь теоретическая работа [20], посвященная этим вопросам, и несколько экспериментальных работ [18-21].

Разработка гиротронов с рабочей частотой в области терагерцового диапазона ведется практически во всех мировых научных центрах, специализирующихся в области вакуумной электроники [22-24]. Так, например, в Массачусетском технологическом институте и компании Communications\&Power Industries созданы непрерывные гиротроны с частотами 0.395 ТГц [25], 0.46 ТГц [26] и 0.527 ГГц [27], а также предложена оригинальная конструкция гироусилителя с рабочей частотой в окрестности 0.25 ТГц [28]. В Институте прикладной физики РАН были разработаны уникальные импульсные соленоиды с индукцией магнитного поля до 50 Тл и на базе этих магнитных систем реализованы гиротроны с длительностью импульса в десятки микросекунд и уровнем мощности в сотни киловатт на частотах $\sim 0.7$ ТГц [29] или киловаттным уровнем мощности на частотах $1-1.3$ ТГц [30]. С использованием не требующих заливки жидким гелием криомагнитов с индукцией магнитного поля до 10-15 Тл созданы непрерывные гиротроны с параметрами выходного излучения 0.263 ГГц (1 кВт) [31], 0.25 ГГц $(200$ кВт) [32] и 0.78 ГГц (10 Вт) [33].

Таким образом, в настоящий момент уже появились субтерагерцовые гиротроны, работающие с достаточно высокой мощностью как в импульсном, так и в непрерывном режимах. Удвоение частоты излучения таких ламп позволит получить узкополосное (несколько МГц) излучение в диапазоне $0.5-1.1$ ТГц с возможностью перестройки частоты излучения в пределах 1 ГГц и в перспективе достичь частоты в 2-2.5 ТГц. При этом большая мощность излучения накачки позволяет рассчитывать на получение высокой мощности излучения на второй гармонике даже при комнатной температуре.

\section{2. Получение второй гармоники в кристаллах InP: Fe}

В данной работе исследована возможность удвоения частоты излучения гиротрона, работающего на основной циклотронной гармонике, с частотой генерации 0.263 ТГц. Как уже было упомянуто, генерация второй гармоники за счет решеточной нелинейности второго порядка возможна только в кристаллах, в которых отсутствует центр инверсии. При этом также важно учитывать дисперсию показателя преломления и поглощение в материале в интересующем диапазоне частот. Действительно, интенсивность излучения на второй гармонике можно вычислить как

$$
I_{2 v}=\frac{128 \pi^{5} \chi^{2} v^{2} L^{2}}{n(v)^{2} n(2 v) c^{3}} I_{v}^{2}\left(\frac{\sin 2(\pi v \Delta n L / c)}{2 \pi v \Delta n L / c}\right)^{2} .
$$

Здесь $v-$ частота, $\chi-$ нелинейная восприимчивость второго порядка, $L$ - длина кристалла, $c$ - скорость света, $n(v)$ - показатель преломления материала, $\Delta n=n(2 v)-n(v)$ [34]. Видно, что эффективность преобразования квадратично зависит от величины нелинейной восприимчивости и длины кристалла при условии, что синусоидальный множитель близок к нулю. Данное 
Величина нелинейной восприимчивости второго порядка в различных материалах $\mathrm{A}^{\mathrm{III}} \mathrm{B}^{\mathrm{V}}$ и $\mathrm{A}^{\mathrm{II}} \mathrm{B}^{\mathrm{VI}}[18,35]$

\begin{tabular}{c|c}
\hline Полупроводник & $\begin{array}{c}\text { Нелинейная } \\
\text { восприимчивость второго } \\
\text { порядка } \chi, 10^{-10} \text { см/B }\end{array}$ \\
\hline $\mathrm{GaAs}$ & 57 \\
$\mathrm{GaP}$ & 18 \\
$\mathrm{GaSb}$ & 150 \\
$\mathrm{InP}$ & 345 \\
$\mathrm{InAs}$ & 155 \\
$\mathrm{CdTe}$ & 100
\end{tabular}

условие соответствует малой дисперсии показателя преломления в материале при увеличении частоты в 2 раза и ограничивает рабочую длину кристалла. Последняя также может быть ограничена разогревом кристалла из-за паразитного поглощения. Как правило, фононные линии поглощения, а также линии поглощения примесей и точечных дефектов в типичных полупроводниковых материалах лежат в области частот > 1 ТГц, поэтому в рассматриваемом спектральном диапазоне основной вклад в поглощение могут вносить потери на свободных носителях, увеличивающиеся степенным образом с ростом длины волны. Несмотря на достаточно высокие значения нелинейной восприимчивости второго порядка (см. таблицу), получение низкой остаточной концентрации свободных носителей представляет собой трудную технологическую задачу для многих из распространенных полупроводников.

Среди материалов, для которых эта проблема успешно решена, следует отметить фосфид индия, в кото-

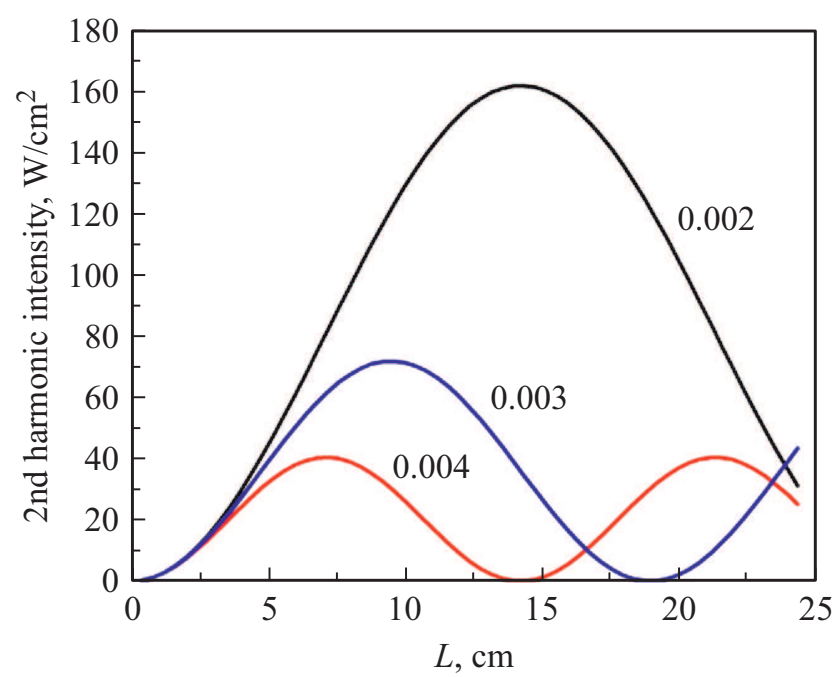

Рис. 1. Рассчитанная интенсивность излучения на удвоенной частоте при возбуждении с интенсивностью 8 кВт/см ${ }^{2}$ на основной частоте 0.263 ТГц в зависимости от длины кристалла $\mathrm{InP}: \mathrm{Fe} L$ при различной дисперсии показателя преломления (значения $\Delta n$ указаны). ром, кроме того, величина нелинейной восприимчивости более чем в 6 раз превышает нелинейную восприимчивость второго порядка в наиболее хорошо исследованном в этом отношении полупроводнике GaAs. Как следует из результатов работы [36], для излучения на частоте 0.263 ТГц разность показателей преломления на первой и второй гармониках $\Delta n=n(2 v)-n(v)$ лежит в диапазоне от 0 до 0.004. На рис. 1 представлен расчет эффективности преобразования для различных значений $\Delta n$ в указанном диапазоне. При расчете предполагалась величина интенсивности излучения на основной частоте $8 \mathrm{\kappa} \mathrm{BT} / \mathrm{cm}^{2}$, что для рассматриваемого гиротрона, работающего на максимальной мощности 1 кВт, соответствует диаметру сфокусированного пучка $\sim 4$ мм. Такая фокусировка может быть легко достигнута на практике [37]. Видно, что даже в случае максимальной дисперсии увеличение рабочей длины кристалла до $7 \mathrm{~cm}$ приводит к росту интенсивности излучения на второй гармонике. Пользуясь коэффициентом поглощения из работы [36], можно оценить мощность, поглощаемую в таком кристалле, которая составляет 50\%, т.е. 500 Вт. Таким образом, используя модуляцию освещения со скважностью $\sim(3-5)$ и простейшую систему активного охлаждения, можно избежать разогрева кристалла в данном режиме.

\section{1. Экспериментальные исследования второй гармоники излучения гиротрона}

Одним из факторов, затрудняющих экспериментальное исследование процесса удвоения частоты гиротрона в полупроводниковых структурах, является присутствие в спектре излучения гиротронов составляющих на гармониках циклотронной частоты [38], обусловленных нелинейностью электронного пучка в режиме сильного сигнала [39]. Для сравнения выполненной оценки эффективности удвоения частоты с интенсивностью собственного излучения гиротрона на второй гармонике были проведены экспериментальные исследования спектра излучения гиротрона с использованием режекторных фильтров на основе циклотронного резонанса в полупроводниковых структурах. Для этого $\sim 10 \%$ излучения гиротрона было отведено в измерительный тракт, состоящий из сверхразмерного волновода, сопряженного с интерферометром Майкельсона или Маха-Цендера, на выходе которого устанавливался закритический волновод, отсекающий излучение на основной гармонике 263 ГГц и пропускающий излучение на удвоенной частоте 526 ГГц. Далее излучение заводилось в волноводную вставку в транспортный сосуд Дьюара СТГ-40, в нижней части которой располагался сверхпроводящий магнит, позволяющий получить магнитное поле до 3 Тл при охлаждении жидким гелием. В качестве режекторного фильтра внутри магнита располагалась структура с квантовой ямой $\mathrm{HgTe} / \mathrm{Cd}_{0.65} \mathrm{Hg}_{0.35}$ Те шириной $20 \mathrm{HM}$, выращенная методом молекулярно-лучевой эпитаксии на подложке из GaAs [40] и легированная индием до 

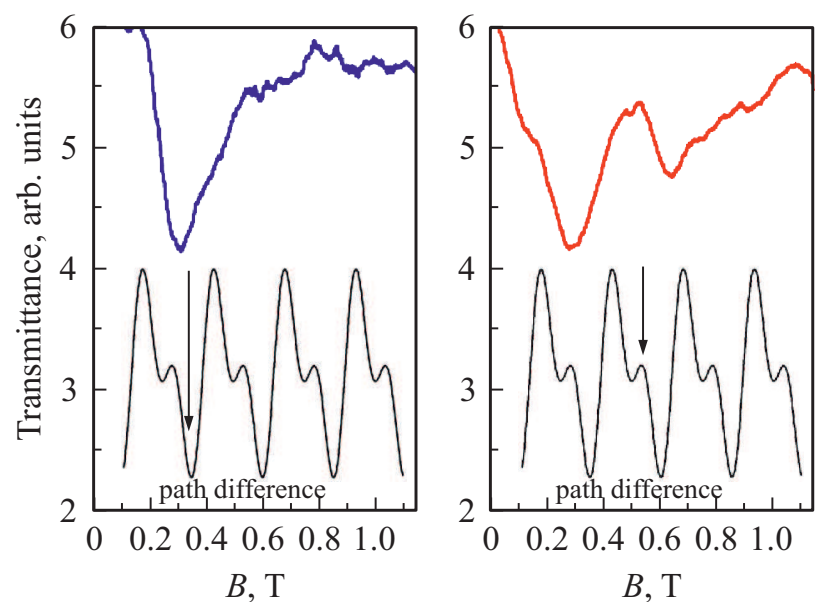

Pис. 2. Спектры магнитопропускания образца на основе $\mathrm{HgCdTe}$ при различных значениях разности хода (отмечены стрелкой на интерферограммах - см. на вставках внизу) в интерферометре Майкельсона, использованном в качестве фильтра второй гармоники.

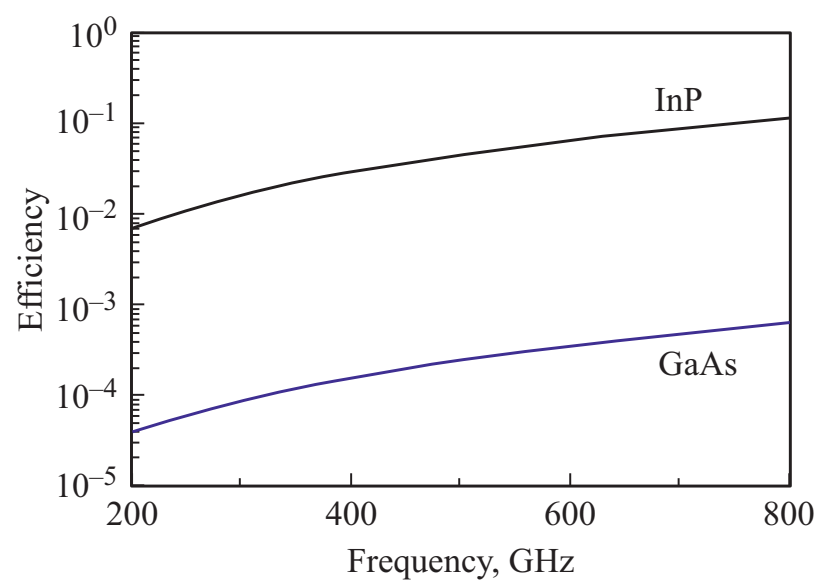

Рис. 3. Рассчитанная эффективность преобразования второй гармоники в различных материалах в зависимости от частоты излучения. При расчетах интенсивность излучения на основной частоте принималась равной $50 \kappa \mathrm{BT} / \mathrm{cm}^{2}$, рабочая длина кристалла 7 см. Параметры для расчета взяты из $[35,36]$.

концентрации $\sim 10^{17} \mathrm{~cm}^{-3}$ в барьерах. Благодаря малым эффективным массам электронов в подобных структурах циклотронный резонанс на частоте 0.263 ТГц наблюдается в относительно небольших магнитных полях, $\sim 0.3$ Тл [41]. При достижении поля, соответствующего циклотронному резонансу, пропускание структуры резко уменьшалось, что приводило к падению сигнала на фотоэлектрическом приемнике на основе InSb, расположенном на конце волноводной вставки за магнитом. Таким образом, описанная измерительная установка позволяла выполнить экспресс-анализ спектрального состава излучения. Величина подавления сигнала на основной гармонике в закритическом волноводе и глубина модуляции в используемых интерферометрах были измерены от- дельно в диапазоне линейности отклика приемника $\mathrm{InSb}$, использовалась возможность непрерывного изменения мощности излучения гиротрона от 1 Вт до 1 кВт. Комбинация интерферометра и закритического волновода позволяла ослабить интенсивность первой гармоники примерно на 3 порядка.

В зависимости величины сигнала на приемнике от разности хода в плечах спектрометра помимо основных максимумов, соответствующих излучению на основной гармонике, были обнаружены слабые максимумы, расположенные между основными. Схематически характер наблюдаемой интерферограммы показан на вставке к рис. 2, где также приведены спектры магнитопропускания, полученные при мощности излучения гиротрона 80 Вт. Видно, что в положении интерферометра, соответствующего минимуму пропускания, спектр магнитопропускания содержит единственную линию циклотронного резонанса, соответствующую частоте излучения гиротрона 0.263 ТГц. При положении интерферометра, соответствующем „дополнительному“ максимуму, в спектре магнитопропускания появляется вторая линия, максимум которой соответствует вдвое большему магнитному полю, т.е. второй гармонике. Измерения при других мощностях гиротрона показали, что среднее отношение интенсивности второй гармоники к первой лежит в диапазоне $0.001-0.005$.

Легко оценить, что для интенсивности излучения на основной гармонике $8 \mathrm{\kappa BT} / \mathrm{cm}^{2}$, рассматриваемой выше, удвоение на кристалле длиной $\sim 7 \mathrm{~cm}$ дает сравнимую интенсивность излучения на второй гармонике. Однако в случае импульсных гиротронов доступны более высокие интенсивности излучения, что позволяет существенно увеличить эффективность преобразования. На рис. 3 приведены сравнительная эффективность преобразования в различных материалах в зависимости от частоты излучения гиротрона при интенсивности на основной гармонике $50 \mathrm{\kappa BT} / \mathrm{cm}^{2}$ и рабочей длине кристалла $7 \mathrm{~cm}$. Следует отметить, что рост эффективности с частотой будет скомпенсирован возрастающей дисперсией преломления и поглощением в InP: Fe, однако при данных параметрах можно рассчитывать на получение интенсивности излучения на удвоенной частоте на порядок больше, чем собственное излучение гиротрона на второй гармонике.

\section{3. Заключение}

В работе приведены оценки и расчеты достижимой интенсивности терагерцового излучения при удвоении частоты на „решеточной“ нелинейности второго порядка в кристаллах $\mathrm{InP}: \mathrm{Fe}$ с использованием субтерагерцовых гиротронов в качестве источника излучения на основной гармонике. Экспериментально показано, что интенсивность излучения, полученного при удвоении частоты, может существенно превышать интенсивность 
собственного излучения гиротрона на второй гармонике. Показана возможность использования излучения на второй гармонике для магнитоспектроскопии полупроводниковых структур.

\section{Финансирование работы}

Работа поддержана грантом Российского научного фонда № 18-79-10112.

\section{Конфликт интересов}

Авторы заявляют, что у них нет конфликта интересов.

\section{Список литературы}

[1] R.E. Miles, P. Harrison, D. Lippens. Terahertz Sources and Systems [NATO Sci. Ser., Ser. II, Vol. 27 (Kluwer Academic Publishers, 2001)].

[2] D. Mittleman. Sensing with Terahertz Radiation [Springer Ser. in Optical Sci., Vol. 85 (Springer-Verlag, 2003)].

[3] D. Vander Weide. Optics \& Photonics News, 14(4), 48 (2003).

[4] Xiong Xu, Yanyu Wei, Fei Shen, Hairong Yin, Jin Xu Yubin Gong, Wenxiang Wang. Phys. Plasmas, 19, 013113 (2012).

[5] B.S. Williams. Nature Photonics, 1, 517 (2007).

[6] S. Fathololoumi, E. Dupont, C.W.I. Chan, Z.R. Wasilewski, S.R. Laframboise, D. Ban, A. Matyas, C. Jirauschek, Q. Hu, H.C. Liu. Opt. Express, 20 (4), 3866 (2012).

[7] X. Wang, C. Shen, T. Jiang, Zh. Zhan, Q. Deng, W. Li, W. Wu, N. Yang, W. Chu, S. Duan. AIP Adv., 6, 075210 (2016).

[8] G.P. Gallerano, S. Biedron. Proc. 2004 FEL Conf. (2004) p. 216.

[9] G. Dodel. Infr. Phys. Technol., 40, 127 (1999).

[10] S.B. Bodrov, A.A. Murzanev, Yu.A. Sergeev, Yu.A. Malkov, A.N. Stepanov. Appl. Phys. Lett., 103, 251103 (2013).

[11] S. Vidal, J. Degert, M. Tondusson, E. Freysz, J. Oberlé. J. Opt. Soc. Am. B, 31, 149 (2014).

[12] T. Tanabe, K. Suto, J. Nishizawa, K. Saito, T. Kimura. Appl. Phys. Lett., 83, 237 (2003).

[13] T. Taniuchi, H. Nakanishi. J. Appl. Phys., 95, 7588 (2004).

[14] R.A. Lewis. J. Phys. D: Appl. Phys., 47, 374001 (2014).

[15] M. Urban, Ch. Nieswand, M.R. Siegrist. J. Appl. Phys., 77, 981 (1995).

[16] F. Keilmann, R. Brazis, H. Barkley, W. Kasparek, M. Thumm, V. Erckmann. Europhys. Lett., 11 (4), 337 (1990).

[17] R. Narkowicz, M.R. Siegrist, Ph. Moreau, J.P. Hogge, R. Raguotis, R. Brazis. Acta Phys. Polon. A, 119 (4), 509 (2011).

[18] A. Mayer, F. Keilmann. Phys. Rev. B, 33, 6954 (1986).

[19] T. Dekorsy, V.A. Yakovlev, W. Seidel, M. Helm, F. Keilmann. Phys. Rev. Lett., 90, 055508 (2003).

[20] J.H. Mc Fee, G.D. Boyd, P.H. Schmidt. Appl. Phys. Lett., 17, 57 (1970).

[21] C. Flytzanis. Phys. Rev. B, 6, 1264 (1972).

[22] S. Alberti, J.-Ph. Ansermet, K.A. Avramides, F. Braunmueller, P. Cuanillon, J. Dubray, D. Fasel, J.P. Hogge, A. Macor, E. De Rijk, M. Da Silva. Phys. Plasmas, 19(12), 123102 (2012).

[23] T. Idehara, M. Glyavin, A. Kuleshov, S. Sabchevski, V. Manuilov, V. Zaslavsky, I. Zotova, A. Sedov. Rev. Sci. Instrum., 88, 094708 (2017).
[24] A.C. Torrezan, M.A. Shapiro, J.R. Sirigiri, R.J. Temkin, R.G. Griffin. IEEE Trans., ED-58 (8), 2777 (2011).

[25] M. Blank, Ph. Borchard, S. Cauffman, K. Felch, M. Rosay, L. Tometich. IEEE Intern. Vacuum Electronics Conf. (IVEC) (Monterey, CA, USA, 2014).

[26] A.C. Torrezan, S.-T. Han, I. Mastovsky, M.A. Shapiro, J.R. Sirigiri, R.J. Temkin, A.B. Barner, R.G. Griffin. IEEE Trans., PS-38 (6), 1150 (2010).

[27] S. Jawla, E. Nanni, M. Shapiro, I. Mastovsky, W. Guss, R. Temkin, R. Griffin. 36th Int. Conf. on Infrared, Millimeter and Terahertz Waves (IRMMW-THz) (Houston, 2011). DOI: 10.1109/irmmw-THz.2011.6105096)

[28] M.Yu. Glyavin, A.G. Luchinin, A.A. Bogdashov, V.N. Manuilov, M.V. Morozkin, Yu. Rodin, G.G. Denisov, D. Kashin, G. Rogers, C.A. Romero-Talamas, R. Pu, A.G. Shkvarunetz, G.S. Nusinovich. Radiophys. Quant. Electron., 56 (8-9), 497 (2014).

[29] E.A. Nanni, S.M. Lewis, M.A. Shapiro, R. Griffin, R. Temkin. Final Progr. 54th Experimental Nuclear Magnetic Resonance Conf. (ENC) Pacific Grove (Snata Clara, 2013) p. 30.

[30] M.Yu. Glyavin, N.S. Ginzburg, A.L. Goldenberg, G.G. Denisov, A.G. Luchinin, V.N. Manuilov, V.E. Zapevalov, I.V. Zotova. Terahertz Sci. Technol., 5(2), 67 (2012).

[31] M.Yu. Glyavin, A.V. Chirkov, G.G. Denisov, A. Fokin, V. Kholoptsev, A.N. Kuftin, A.G. Luchinin, G. Golubiatnikov, V. Malygin, M. Morozkin, V. Manuilov, M. Proyavin, A. Sedov, E.V. Sokolov, E.M. Tai, A. Tsvetkov, V.E. Zapevalov. Rev. Sci. Instrum., 86, 054705 (2015).

[32] G.G. Denisov, M.Yu. Glyavin, A.P. Fokin,A.N. Kuftin, A.I. Tsvetkov, A.S. Sedov, E.A. Soluyanova, M.I. Bakulin, E.V. Sokolov, E.M. Tai, M.V. Morozkin, M.D. Proyavin, V.E. Zapevalov. Rev. Sci. Instrum., 89 (8), 084702 (2018).

[33] T. Idehara, M. Glyavin, A. Kuleshov, S. Sabchevski, V. Manuilov, V. Zaslavsky, I. Zotova, A. Sedov. Rev. Sci. Instrum., 88, 094708 (2017).

[34] F. Zernike, J.E. Midwinter. Applied Nonlinear Optics (John Wiley \& Sons Inc., 1973).

[35] O. Madelung. Semiconductors: Data Handbook (Springer Verlag, N. Y., 2003).

[36] L.N. Alyabyeva, E.S. Zhukova, M.A. Belkin, B.P. Gorshunov. Sci. Rep., 7 (1), 7360 (2017).

[37] A.V. Vodopyanov, A.V. Samokhin, N.V. Alexeev, M.A. Sinayskiy, A.I. Tsvetkova, M.Yu. Glyavin, A.P. Fokin, V.I. Malygin. Vacuum, 145, 340 (2017).

[38] G.Yu. Golubiatnikov, M.A. Koshelev, A.I. Tsvetkov, A.P. Fokin, M.Yu. Glyavin, M.Yu. Tretyakov. EPJ Web Conf., 195, 06017 (2018).

[39] N.A. Zavol'skii, G.S. Nusinovich, A.B. Pavel'ev. Radiophysics Quantum Electron., 31 (3), 269 (1988).

[40] N.N. Mikhailov, R.N. Smirnov, S.A. Dvoretsky, Yu.G. Sidorov, V.A. Shvets, E.V. Spesivtsev, S.V. Rykhlitski. Int. J. Nanotechnol., 3 (1), 120 (2006).

[41] A.V. Ikonnikov, A.A. Lastovkin, K.E. Spirin, M.S. Zholudev, V.V. Rumyantsev, K.V. Maremyanin, A.V. Antonov, V.Ya. Aleshkin, V.I. Gavrilenko, S.A. Dvoretskii, N.N. Mikhailov, Yu.G. Sadofyev, N. Samal. JETP Lett., 92 (11), 756 (2010).

Редактор Л.В. Шаронова 


\title{
Second harmonic generation of subterahertz gyrotron radiation by frequency doubling in InP : Fe and its application for magnetospectroscopy of semiconductor structures
}

V.V. Rumyantsev ${ }^{1}$, K.V. Maremyanin ${ }^{1}$, A.P. Fokin ${ }^{2}$, A.A. Dubinov ${ }^{1}$, V.V. Utochkin ${ }^{1}$, M.Yu. Glyavin ${ }^{2}$, N.N. Mikhailov ${ }^{3}$, S.A. Dvoretsky ${ }^{3}$, S.V. Morozov ${ }^{1}$, V.I. Gavrilenko ${ }^{1}$

${ }^{1}$ Institute for Physics of Microstructures, Russian Academy of Sciences, 603950 Nizhny Novgorod, Russia

${ }^{2}$ Institute of Applied Physics, Russian Academy of Sciences, 603950 Nizhny Novgorod, Russia ${ }^{3}$ Institute for Semiconductor Physics, Siberian Branch of Russian Academy of Sciences, 630090 Novosibirsk, Russia

\begin{abstract}
We discuss the possibility of generating terahertz radiation with high intensity exploiting the second order lattice nonlinearity in indium phosphide crystals doped with iron. Subterahertz gyrotrons were used as a radiation source at a fundamental frequency. The efficiency of the frequency conversion is shown to be as high as 3\%, which opens prospects for developing a new generation of terahertz radiation sources. The possibility of applying second harmonic radiation for magnetospectroscopy of semiconductor structures with quasi-Dirac dispersion is demonstrated.
\end{abstract}

